\title{
DC and AC Shielding Properties of Bulk High-Tc Superconducting Tubes
}

\author{
Jean-Francois Fagnard, Samuel Denis, Gregory Lousberg, Michel Dirickx, Marcel Ausloos, \\ Benoit Vanderheyden, and Philippe Vanderbemden
}

\begin{abstract}
We have studied numerically and experimentally the magnetic flux penetration in high- $T_{c}$ superconducting tube subjected to a uniform magnetic field parallel to its long axis. This study is carried in view of designing low-frequency magnetic shields by exploiting the diamagnetic properties of high- $T_{c}$ superconducting ceramics. We have measured the field attenuation for applied magnetic fields in the frequency range $5 \mathrm{mHz}-0.1 \mathrm{~Hz}$ by Hall probe measurements and at audio frequencies using a sensing coil. A simple 1D analysis using the Kim critical state model was found to be able to reproduce the experimental data satisfactorily. We have also determined the phase shift between the internal and the applied field both experimentally and numerically. Finally, we have studied the sweep rate dependence of the magnetic shielding properties, using data recorded either at several constant sweep rates $\mathrm{d} B / \mathrm{d} t$ or at several $A C$ fields of various amplitudes and frequencies. Both methods agree with each other and lead to a $n$-value of the $E \sim J^{\mathrm{n}}$ law equal to $\sim 40$ at $77 \mathrm{~K}$.
\end{abstract}

Index Terms-Bi-2223 cylinder, magnetic shielding.

\section{INTRODUCTION}

D UE to their ability to expel magnetic flux, superconducting materials have a significant potential to act as efficient magnetic shields [1]-[9]. The advantage of superconductors over traditional approaches is essentially marked at very low frequencies $(f<1 \mathrm{kHz})$, where the magnetic shielding properties of non-magnetic metallic materials - exploiting the skin effect- become inefficient. If low temperatures are allowed by the application (e.g. cooling with liquid nitrogen at 77 $\mathrm{K}$ ), high-temperature superconductors (HTS) can compete with soft ferromagnetic materials, both in terms of shielding factor $S F$ (ratio of the applied field over the magnetic field inside the shield) and of threshold induction $B_{\text {lim }}$, i.e. the maximum induction that can be strongly attenuated by the shield [1], [2].

Manuscript received August 25, 2008. First published June 30, 2009; current version published July 15, 2009.

J.-F. Fagnard, G. Lousberg, B. Vanderheyden, and P. Vanderbemden are with the SUPRATECS and Department of Electrical Engineering and Computer Science B28, Sart-Tilman, B-4000 Liège, Belgium (e-mail: fagnard@ montefiore. ulg.ac.be; gregory.lousberg@ulg.ac.be; B.Vanderheyden@ulg.ac.be; Philippe. Vanderbemden@ulg.ac.be).

S. Denis and M. Dirickx are with the SUPRATECS, Communication, Information Systems \& Sensors (CISS) Department, Royal Military Academy of Belgium, B-1000 Bruxelles, Belgium (e-mail: sdenis@ulg.ac.be; Michel.Dirickx@rma.ac.be).

M. Ausloos is with the SUPRATECS and Physics Institute B5, University of Liège, Sart-Tilman, B-4000 Liège, Belgium (e-mail: Marcel.Ausloos@ulg.ac. be).

Color versions of one or more of the figures in this paper are available online at http://ieeexplore.ieee.org.

Digital Object Identifier 10.1109/TASC.2009.2017880
A number of results can be found in the literature on the magnetic shielding properties of bulk polycrystalline tubes [5], [6], [8]. In our previous work in particular [1], [2], we have studied the magnetic shielding of a Bi-2223 polycrystalline tube subjected to an axial field. Experimental data have been confronted with a computer modeling based on the numerical model developed by Brandt [10]. In the present work, we aim at extending this set of investigations through carrying out magnetic shielding measurements in various experimental configurations ("DC" and "AC" regimes). In particular we will compare results obtained for several sweep rates of the applied DC field $\mathrm{d} H_{a p p} / \mathrm{d} t$ and several frequencies of the applied AC field $H_{a p p}=H_{0} \sin (\omega \mathrm{t})$. The parameters extracted from the measurements will be used to determine precisely the amplitude first harmonic of the internal magnetic field (i.e. inside the superconducting tube) and its phase-lag with respect to the applied magnetic field. We will examine the validity of a simple Bean-Kim model to analyse the data and we will verify the existence of scaling laws.

\section{EXPERIMENT}

The studied sample is a $8 \mathrm{~cm}$ long commercial $\mathrm{Pb}$-doped Bi-2223 polycrystalline cylinder from CAN Superconductors (CST-12/80). The inner diameter of the tube is $12 \mathrm{~mm}$ and the thickness of the wall is $1.6 \mathrm{~mm}$. All measurements are carried out at $T=77 \mathrm{~K}$ and the sample is always cooled down in zero field. Two experimental setups are used for characterizing the magnetic shielding properties of the tube.

In the first setup, a high sensitivity Hall probe (Arepoc HHP-MP) is used to measure the axial magnetic induction inside the superconducting tube. The sensor dimensions are $7 \mathrm{~mm} \times 5 \mathrm{~mm} \times 1 \mathrm{~mm}$. The Hall probe voltage is connected at the input of an instrumentation amplifier with a $30 \mathrm{~Hz}$ filter stage prior to being digitized by a National Instrument Data Acquisition Card (DAQ). An axial DC applied magnetic field up to $36 \mathrm{mT}$ is generated by a solenoidal magnet connected to a HP6030A DC power supply connected to a computer-controlled switch for double-polarity operation. A Labview interface allows us to apply well-definite applied field vs. time waveforms. In the present work, triangular and sinusoidal waveforms are used with frequencies ranging from $2.5 \mathrm{mHz}$ to $0.1 \mathrm{~Hz}$. The whole setup is placed inside a mu-metal ferromagnetic enclosure in order to provide a shielding of the external magnetic fields (including Earth's field).

In a second setup, an axial AC applied magnetic field is generated by a long solenoid connected to an $\mathrm{AC}$ multifunction synthesizer (HP8904A) followed by an audio D-class amplifier (Crown XTi 2000). A LC low-pass filter (cut - off frequency $=10 \mathrm{kHz}$ ) is placed at the output of the 


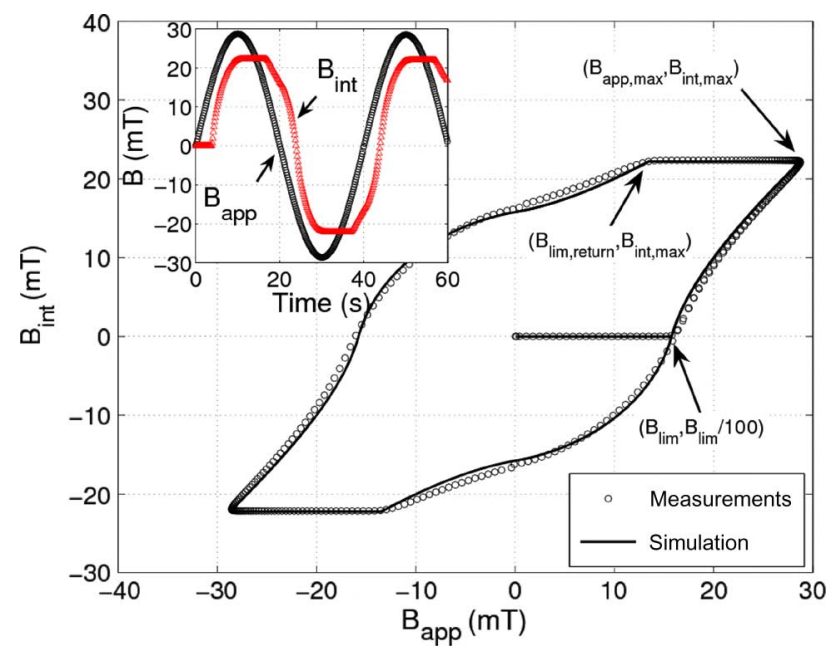

Fig. 1. Hysteretic curve of Bi-2223 tube at $25 \mathrm{mHz}$ and with $B_{\max }=$ 27.8 mT. Circles: measurement data, line: Bean-Kim model results. Inset: Applied and internal magnetic flux densities vs. time (experimental).

amplifier to reject the high frequency parasitic signals generated by the amplifier itself. The measurements are performed in the frequency range $27-507 \mathrm{~Hz}$. The maximum applied magnetic induction is $40 \mathrm{mT}$ (RMS) at $27 \mathrm{~Hz}$ and $14.5 \mathrm{mT}$ (RMS) at $507 \mathrm{~Hz}$. The magnetic field inside the superconducting tube is probed with a miniature coil ( 800 turns $-5 \mathrm{~mm}$ length) wound on a $5 \mathrm{~mm}$ diameter Pyrex rod. The induced electro-motive force (e.m.f.) is applied to a EG\&G 7260 Lock-In Amplifier sensitive to the 1st harmonic of the signal. For all the applied magnetic fields, the amplitude of the reference signal is adjusted exactly to $1 \mathrm{~V}$ RMS in order to minimize the phase shift caused by the Lock-In Amplifier itself. The minimum magnetic induction that can be detected with this setup is around $1 \mathrm{nT}$ at $103 \mathrm{~Hz}$.

\section{RESULTS AND DISCUSSION}

\section{A. Hysteresis and Determination of $J_{c}(B)$ Parameters}

Using the setup based on Hall probe measurements, a very low frequency ( $\mathrm{f}<0.1 \mathrm{~Hz}$ ) sinusoidal magnetic field $H_{a p p}(t)=H_{\max } \sin (\omega t)$ is applied to the tube. The internal magnetic flux density vs. time $B_{\text {int }}(t)$ is sampled at high rate with the DAQ. The data are used to determine $B_{\text {int }}$ as a function of $B_{a p p}=\mu_{0} H_{a p p}$. The as-obtained hysteresis cycle for an amplitude $\mu_{0} H_{\max }=27.8 \mathrm{mT}$ amplitude and a frequency $f=25 \mathrm{mHz}$ is shown in Fig. 1. At low increasing fields starting from $B_{a p p}=0$, the magnetic shielding is effective and the internal induction is below the sensitivity of our experimental setup $\left(B_{\text {int }} \approx 0\right)$ up to some threshold value $B_{\text {lim }}$. Above $B_{\text {lim }}$, the shielding currents cannot prevent the magnetic field from entering in the internal volume of the cylinder and the internal induction increases up to some value $B_{\text {int, } \max }$. On reversal of the applied magnetic field starting from the maximum value $\left(B_{a p p}=B_{\max }\right)$, the internal magnetic flux density remains constant until a value that is called $B_{\text {lim, return }}$ is reached and then it follows hysteretic curve similar to what can be expected for an irreversible type-II superconductor [1], [2], [6].

In the case of a cylinder of finite length, the applied magnetic field can penetrate inside the volume of the tube using two routes: from the external surface of the cylinder and from the opening ends. A previous 2D computer modeling of the magnetic field distribution [1] on a cylinder of the same geometry using the model based on the Brandt algorithm [10] has shown that the behavior of the internal field measured at the centre of the tube is mainly dictated by the penetration from the external surface of the tube and not from the open ends. Such behavior is due to the relatively large aspect ratio (length over radius ratio) of the studied sample. It is therefore of interest to model the superconducting properties shown in Fig. 1 using a simple analytical 1D model [11]. We assume an infinite cylinder characterized by a $J_{c}(B)$ following the Kim law [12]: $J_{c}=J_{c 0}\left(1+|B| / B_{1}\right)^{-1}$. Using this model for a superconducting tube of thickness $d$, the parameters $B_{\text {lim }}$ and $B_{\text {int, } \max }$ can be analytically expressed as a function of $B_{\max }, B_{1}$ and $B_{0}=\mu_{0} d J_{c 0}$ through the relations

$$
\begin{aligned}
B_{\lim } & =-B_{1}+\sqrt{B_{1}^{2}+2 d \mu_{0} J_{c 0} B_{1}} \\
B_{\text {int }, \text { max }} & =-B_{1}+\sqrt{\left(B_{1}+B_{\max }\right)^{2}-2 d \mu_{0} J_{c 0} B_{1}} .
\end{aligned}
$$

It is therefore possible to extract the $J_{c 0}$ and $B_{1}$ parameters-i.e. the whole $J_{c}(B)$-from the measured values of $B_{l i m}$ and $B_{\text {int, } \max }$ using the relations

$$
\begin{aligned}
& B_{1}=\frac{1}{2} \frac{\left(B_{\text {lim }}^{2}+B_{\text {int }, \text { max }}^{2}-B_{a p p, \max }^{2}\right)}{\left(B_{\text {app }, \text { max }}-B_{\text {lim }}-B_{\text {int }, \text { max }}\right)} \\
& B_{0}=d \mu_{0} J_{c 0}=B_{\text {lim }}\left(1+\frac{B_{\text {lim }}}{2 B_{1}}\right) .
\end{aligned}
$$

The analytical model predicts that the internal flux density $B_{\text {int }}\left(B_{a p p}=0\right)$ in the decreasing branch should be equal to $-B_{\text {lim }}$. In practice, a small difference exists $\left(B_{i n t}\left(B_{a p p}=\right.\right.$ $0)=16.4 \mathrm{mT}$ and $B_{\text {lim }}=15.8 \mathrm{mT}$ ). The $B_{\text {lim }}$ values used for extracting $B_{1}$ and $J_{c 0}$ parameters correspond to the mean of measured $B_{\text {lim }}$ and $B_{\text {int }}\left(B_{a p p}=0\right)$ values. The resulting simulation curve simulated with $J_{c 0}=2.310^{7} \mathrm{~A} / \mathrm{m}^{2}$ and $B_{1}=4.5 \mathrm{mT}$ is plotted as a straight line in the Fig. 1: the agreement between the simulation and the measured data is very satisfactory. The method mentioned above does not require curve fitting and allows to extract the $J_{c}(B)$ parameters in a simple manner.

The inset of the Fig. 1 shows the time evolution of both the applied and the internal magnetic fields. The non-linear and hysteretic behavior shown in Fig. 1 translates into a distorted waveform. The first harmonic displays a phase-lag with respect to $B_{a p p}(t)=B_{\max } \sin (\omega t)$. This phase-lag is analyzed in the following paragraphs.

Contrarily to the results that could be obtained using a power-law $E(J)$ model [1], [2], [6], [10], the Bean model does not predict any frequency dependence of the measured signals. In practice however, $B_{\text {int }}$ vs. $B_{a p p}$ measured curves are slightly frequency-dependent. We use (3) and (4) to extract the pair of parameters $J_{c 0}$ and $B_{1}$ from measurements carried out at several frequencies ranging between $5 \mathrm{mHz}$ and $0.1 \mathrm{~Hz}$.

With these parameters determined for each frequency, we calculate analytically the $B_{\text {int }}(t)$ curves for several applied field amplitudes $B_{\max }$. The amplitude, $B_{1 s t}$, and the phase shift, $\theta_{1 s t}$, of its first harmonic of the internal field can be determined, and results compared to experimental data. Results are plotted in 

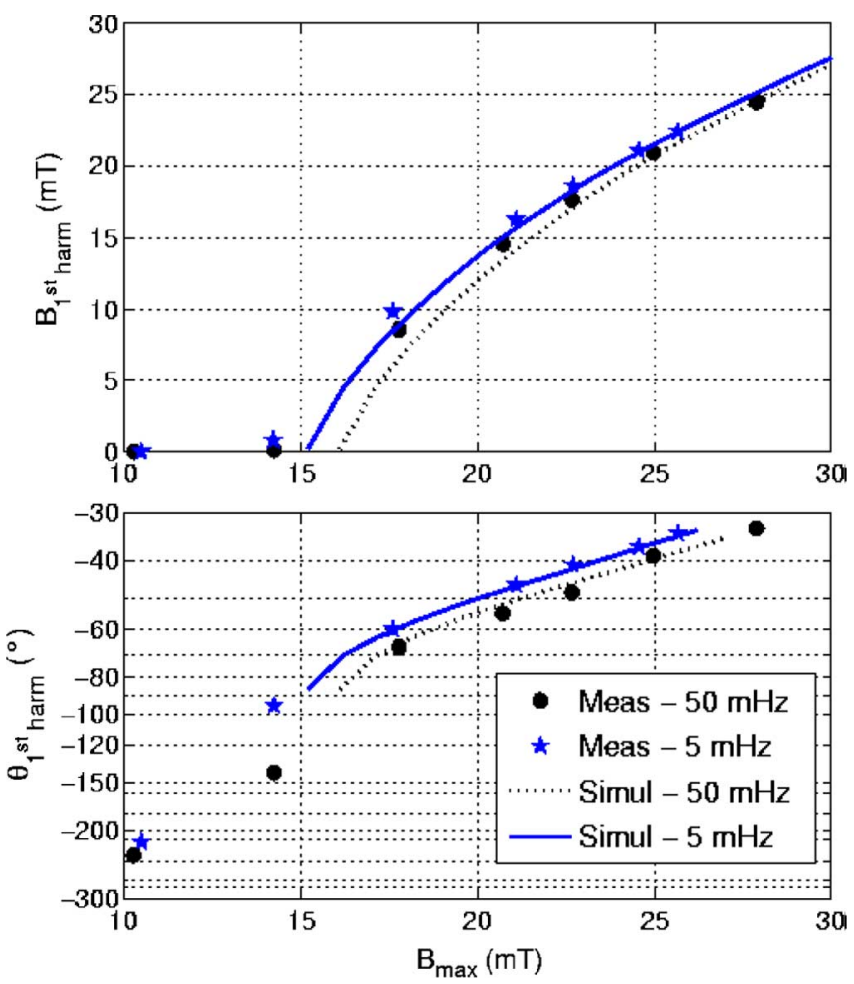

Fig. 2. Amplitude and phase shift of the 1st harmonic of the internal field vs amplitude of the applied magnetic flux density at $5 \mathrm{mHz}$ and $50 \mathrm{mHz}$. Symbols: measurements data, lines: Bean-Kim model results.

Fig. 2, showing the amplitude (top) and the phase lag (bottom) of first harmonic of $B_{\text {int }}$ for increasing amplitudes of magnetic induction at $f=5$ and $50 \mathrm{mHz}$. The lines represent the curves obtained by simulations using $J_{c 0}$ and $B_{1}$ calculated from experimental hysteretic loop at these frequencies for $B_{\max }=27 \mathrm{mT}$. The symbols represent the amplitude and the phase shift of the $B_{\text {int }}$ first harmonic determined from experimental data at these frequencies and different maximum amplitudes between 10 and $26 \mathrm{mT}$.

At amplitudes smaller than $B_{\text {lim }}$, the amplitude and the phase shift cannot be calculated analytically because the model predicts a perfect shielding $\left(B_{\text {int }}=0\right)$, unlike modeled data using a power-law for $E(J)$ as in [6]. Nevertheless, at applied fields below $B_{l i m}$, the internal magnetic field is experimentally measurable and the results are plotted in Fig. 2. For $B>B_{\text {lim }}$, the agreement between measurements and calculation results is very satisfactory in the investigated frequency range. Therefore a simple $1 \mathrm{D}$ analysis using the Bean-Kim model seems to be appropriated for determining the $J_{c}(B)$ characteristics from experimental data and to predict the magnetic behavior of the tube.

\section{B. Flux Creep Effects and Determination of $n$}

The analysis carried out above does not allow us to analyse the sweep rate dependence of the magnetic properties due to flux creep effects. In this section we aim at using the experimental data for determining the $n$-value of the $E(J)$ power law $E(J)=E_{c}\left(J / J_{c}\right)^{n}$. We have therefore measured the threshold induction $B_{\text {lim }}$ for different sweep rates $\mathrm{d} B_{\text {app }} / \mathrm{d} t$. In our experiment, the applied magnetic field is ramped linearly up to $30 \mathrm{mT}$ at constant sweep rates ranging between $0.02 \mathrm{mT} / \mathrm{s}$ and $20 \mathrm{mT} / \mathrm{s}$. In view of an unambiguous determination of $B_{\text {lim }}$ for

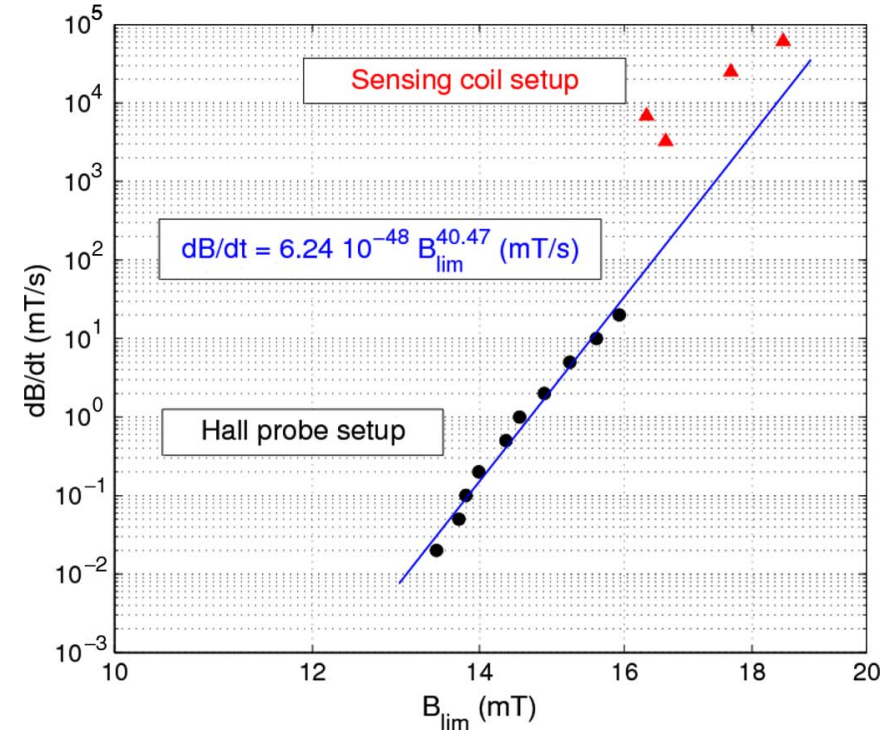

Fig. 3. Measurement of $\mathrm{d} B_{a p p} / \mathrm{d} t$ vs. $B_{l i m}$ with different experimental setups.

each sweep rate, we define $B_{l i m}$ as the applied magnetic induction above which the dimensionless shielding factor, SF (ratio of the applied over the internal magnetic field) becomes smaller than 100.

The results of the $B_{\text {lim }}$ dependence with respect to $\mathrm{d} B_{\text {app }} / \mathrm{d} t$ are shown in Fig. 3. Note that a log-log scale is used. In order to facilitate further interpretation, the graph axes are reversed and show $\mathrm{d} B_{a p p} / \mathrm{d} t$ vs. $B_{\text {lim }}$. In these axes, measurements data points are aligned and we can thus reasonably infer a power law dependence. This result can be understood as follows. For an infinite cylinder of external radius $R$ subjected to a timevarying magnetic field, the average electric field is given by $E=$ $(R / 2) .\left(\mathrm{d} B_{a p p} / \mathrm{d} t\right)[13]$. The value of " $B_{\text {lim }}$ " corresponds to a full penetration of the magnetic flux in the cylindrical sample wall, and is therefore proportional to the average current density $\langle J\rangle$ flowing in the sample. Since $\mathrm{d} B / d \mathrm{t} \propto\langle E\rangle$ and $B_{\text {lim }} \propto$ $\langle J\rangle$, it follows that the exponent of the $\mathrm{d} B / \mathrm{d} t$ vs. $B_{\text {lim }}$ power law should correspond to the $n$ value of $E(J)$.

By fitting our measurements, we find $n=40.5$. This value is substantially higher than those reported elsewhere on Bi-2223 materials [14]-[16]. We explain it by considering the fact that the non-textured polycrystalline Bi-2223 materials studied here displays a strongly field-dependent $J_{c}$. If we approximate this dependence by a power-law: $J_{c}=J^{*}\left(|B| / B^{*}\right)^{-\gamma}$ in the range of magnetic fields used, we find a $\gamma$-value of 0.65 . For a material characterized by a power-law: $E=E_{c}\left(J / J_{c}\right)^{n}$ and a field dependent $J_{c}\left(B^{-\gamma}\right)$, the $J_{c}$ field dependence affects the magnetic relaxation in such a way that the magnetic properties are equivalent to those of a material characterized by a constant $J_{c}$, and with an apparent exponent $n^{\prime}$ given by $n^{\prime}=n(1+\gamma)$ [14]. Taking $n^{\prime}=40.5$ and $\gamma=0.65$, we find $n=24.2$, which is in agreement with values reported in the literature [1], [14]-[16].

The sensing coil based experimental set-up based is used to measure the shielding properties with AC fields $H_{a p p}(t)=$ $H_{0} \sin (2 \pi f \mathrm{t})$ at audio frequencies $(27 \mathrm{~Hz}<f<507 \mathrm{~Hz})$, in order to extend the range of sweep rates and to see whether the power-law behavior mentioned above still holds true. In 


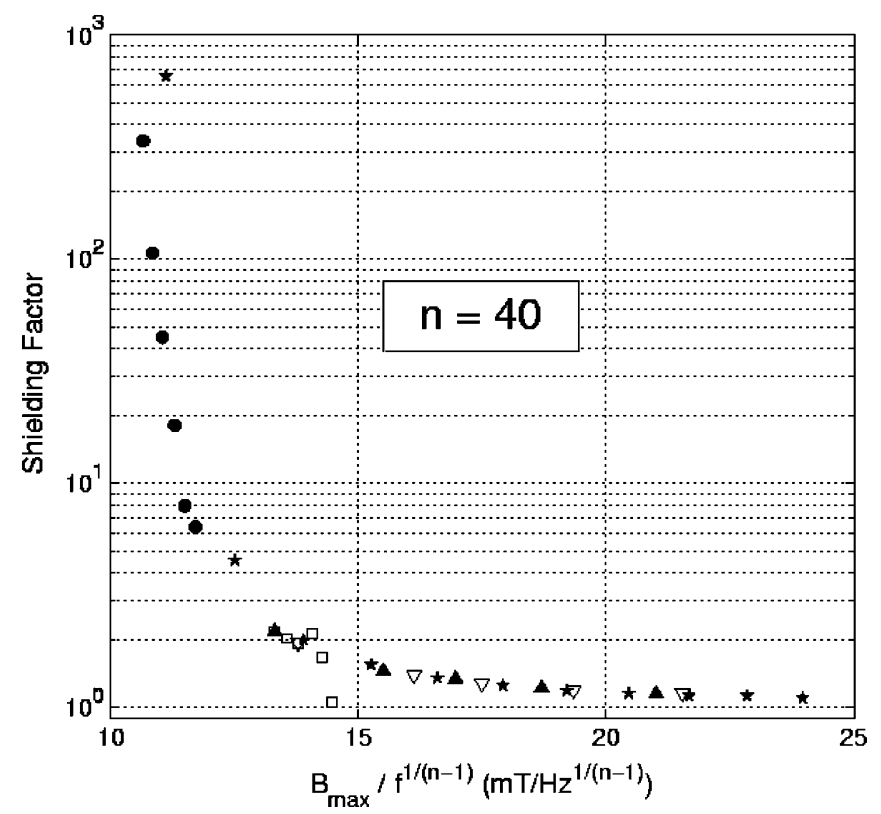

Fig. 4. Shielding factor measured for at several frequencies and $B_{\max }$ and plotted in function of a single variable $B_{\max }, / f^{1 /(n-1)}$.

view of comparing the $\mathrm{AC}$ data with those obtained with the field ramped at a constant sweep rate, we take as an average $\mathrm{d} B_{a p p} / \mathrm{d} t$ the maximum applied flux density divided by the quarter of the period, i.e. $4 f \mu_{0} H_{0}$. As can be seen in Fig. 3, the measurements at $27 \mathrm{~Hz}, 207 \mathrm{~Hz}$ and $507 \mathrm{~Hz}$ are in good agreement with the previously obtained dependence represented by a plain line.

Another way of determining the exponent $n$ from measurements is to use scaling laws [1], [2], [10], [17]. The AC susceptibility $\left(\chi=\chi^{\prime}-j \chi^{\prime \prime}\right)[17]$ and hence the shielding factor SF measured for several combinations of $B_{\max }$, and $f$ should be a factor of a single variable $B_{\max } / f^{1 /(n-1)}$. On Fig. 4, several data sets obtained with various $B_{\max }$ ranging between 14.2 and $33.6 \mathrm{mT}$ and $f$ between 2.5 and $100 \mathrm{mHz}$ are plotted with respect to the this single variable using a $n$-value of 40 as it has been determined on the fit presented on Fig. 3. It can be seen that all the experimental data points scale on a single curve. This result shows that the $n$-value can also be determined from SF of the tube measured at sinusoidal applied fields at several frequencies/amplitudes and that the results agree with those obtained with applied fields ramped linearly (i.e. at constant $\mathrm{d} B / \mathrm{d} t$ ).

\section{CONCLUSION}

The shielding properties of a bulk polycrystalline Bi-2223 tube in axial magnetic field configuration have been investigated both experimentally with two different experimental setups and numerically by using a simple Bean-Kim model. This model allowed us to extract intrinsic parameters of the $J_{c}(B)$ dependence from experimental hysteresis curve and to use them to numerically compute the amplitude and phase of the first harmonic of the internal magnetic field. The agreement between simulations and experimental results was found to be very good.

The $B_{\text {lim }}$ dependence on the sweep rate of the magnetic field was studied and the results showed power law dependence with an exponent of 40.5. This exponent can be related to the non linearity of the electrical field by considering the Maxwell laws and the Bean model. The value of this exponent is higher than those reported in the literature but can be explained by the strong field dependence of the critical current density. The $E(J)$ power law leading to scaling laws that has been verified by plotting a large number of results for several $B_{a p p}$ and $f$.

\section{ACKNOWLEDGMENT}

The authors thank the Royal Military Academy, FNRS and ULg for cryofluid, equipment and travel grants.

\section{REFERENCES}

[1] S. Denis, L. Dusoulier, M. Dirickx, P. Vanderbemden, R. Cloots, M. Ausloos, and B. Vanderheyden, "Magnetic shielding properties of high-temperature superconducting tubes subjected to axial fields," Supercond. Sci. Technol., vol. 20, pp. 192-201, 2007.

[2] S. Denis, M. Dirickx, P. Vanderbemden, M. Ausloos, and B. Vanderheyden, "Field penetration into hard type-II superconducting tubes: Effects of a cap, a non-superconducting joint, and non-uniform superconducting properties," Supercond. Sci. Technol., vol. 20, pp. 418-427, 2007.

[3] J. R. Clem, "Zero-quantum superconducting magnetic shielding apparatus and method," IEEE Trans. Magn., vol. 19, pp. 1278-1281, 1983.

[4] F. Pavese, "Magnetic shielding," in Handbook of Applied Superconductivity, B. Seeber, Ed. London: Taylor \& Francis, 1998, pp. $1461-1483$.

[5] V. Plechacek, E. Pollert, and J. Hejtmanek, "Influence of the microstructure on magnetic-shielding properties of $(\mathrm{Bi}, \mathrm{Pb})-\mathrm{Sr}-\mathrm{Ca}-\mathrm{Cu}-\mathrm{O}$ superconductor," Materials Chemistry and Physics, vol. 43, pp. 95-98, 1996.

[6] F. Sirois, J. R. Cave, and Y. Basile-Bellavance, "Non-linear magnetic diffusion in a Bi2212 hollow cylinder: Measurements and numerical simulations," IEEE Trans. Appl. Supercond., vol. 17, pp. 3652-3655, 2007.

[7] T. Cavallin, R. Quarantiello, A. Matrone, and G. Giunchi, "Magnetic shielding of $\mathrm{MgB}_{2}$ tubes in applied DC and AC field," Journal of Physics: Conference Series, vol. 43, pp. 1015-1018, 2006.

[8] H. Matsuba, A. Yahara, and D. Irisawa, "Magnetic shielding properties of HTc superconductor," Supercond. Sci. Technol., vol. 5, pp. S432-S439, 1992.

[9] C. Navau, A. Sanchez, E. Pardo, D.-X. Chen, E. Bartolomé, X. Granados, T. Puig, and X. Obradors, "Critical state in finite type-II superconducting rings," Phys. Rev. B, vol. 71, pp. 214507-214509, 2005.

[10] E. H. Brandt, "Superconductor disks and cylinders in an axial magnetic field. I. Flux penetration and magnetization curves," Phys. Rev. B, vol. 58, pp. 6506-6522, 1998.

[11] C. P. Bean, "Magnetization of hard superconductors," Phys. Rev. Lett., vol. 8, pp. 250-253, 1962.

[12] Y. B. Kim, C. F. Hempstead, and A. R. Strnad, "Critical persistent currents in hard superconductors," Phys. Rev. Lett., vol. 9, pp. 306-309, 1962.

[13] D. Caplin, L. F. Cohen, G. K. Perkins, and A. A. Zhukov, "The electric field within high-temperature superconductors: Mapping the E-J-B surface," Supercond. Sci. Technol., vol. 7, pp. 412-422, 1994.

[14] P. Vanderbemden, Z. Hong, T. A. Coombs, S. Denis, M. Ausloos, J. Schwartz, I. B. Rutel, N. H. Babu, D. A. Cardwell, and A. M. Campbell, "Behavior of bulk high-temperature superconductors of finite thickness subjected to crossed magnetic fields: Experiment and model," Phys. Rev. B, vol. 75, pp. 174515-174514, 2007.

[15] M. Dhallé, M. Cuthbert, M. D. Johnston, J. Everett, R. Flükiger, S. X. Dou, W. Goldacker, T. Beales, and A. D. Caplin, "Experimental assessment of the current-limiting mechanisms in BSCCO/Ag hightemperature superconducting tapes," Supercond. Sci. Technol., vol. 10, pp. 21-31, 1997.

[16] H. Yamasaki and Y. Mawatari, "Current-voltage characteristics and flux creep in melt-textured YBa2Cu3O7- $\delta$," Supercond. Sci. Technol., vol. 13, pp. 202-208, 2000.

[17] D.-X. Chen, A. Sanchez, C. Navau, Y.-H. Shi, and D. A. Cardwell, "Critical-current density of melt-grown single-grain Y-Ba-Cu-O disks determined by ac susceptibility measurements," Supercond. Sci. Technol., vol. 21, pp. 085013-085016, 2008. 